\title{
Cipango
}

Cahiers d'études japonaises

\section{La question de la conscription au Japon après l'adoption des nouvelles lois japonaises sur la sécurité nationale : propos sur un fantasme récurrent}

Military service in Japan after the adoption of the new laws on national security: considerations on a recurrent fantasy

Éric Seizelet

\section{OpenEdition}

\section{Journals}

Édition électronique

URL : https://journals.openedition.org/cipango/4998

DOI : 10.4000/cipango.4998

ISSN : 2260-7706

Éditeur

INALCO

Édition imprimée

Date de publication : 20 octobre 2021

Pagination : 277-315

ISBN : 9782858313969

ISSN : $1164-5857$

Référence électronique

Éric Seizelet, "La question de la conscription au Japon après l'adoption des nouvelles lois japonaises sur la sécurité nationale : propos sur un fantasme récurrent », Cipango [En ligne], 24 | 2021, mis en ligne le 11 octobre 2021, consulté le 10 avril 2022. URL : http://journals.openedition.org/cipango/4998 ; DOI : https://doi.org/10.4000/cipango.4998

\section{(c) (†) 8}

Cipango est mis à disposition selon les termes de la Licence Creative Commons Attribution - Pas d'Utilisation Commerciale 4.0 International. 


\section{La question de la conscription au Japon après l'adoption des nouvelles lois japonaises sur la sécurité nationale : propos sur un fantasme récurrent}

Military service in Japan after the adoption of the new laws
on national security: considerations on a recurrent fantasy

Éric Seizelet

Université Paris-Diderot

\section{Introduction}

En février 2012, un tweet du vice-gouverneur de Tōkyō, Inose Naoki 猪瀬 直樹, soulignait que les opérations d'assainissement de la centrale nucléaire de Fukushima ne concernaient pas seulement les sous-traitants de l'opérateur Tōden 東京電力, mais que cette 《guerre » devait être prise à bras le corps par l'ensemble des citoyens. Aussitôt, les réseaux sociaux se sont mobilisés pour dénoncer la « conscription nucléaire ». Un an plus tard, dans une interview télévisée en date du mois d'avril 2013, le secrétaire général (kanjichō 幹事長) du Parti libéral-démocrate (PLD, jiyn̂ minshutô 自由民主党) Ishiba Shigeru石破 茂 - un ancien des Forces d'autodéfense (FAD, Jieitai自衛隊), connu pour ses positions hétérodoxes sur la conscription par rapport à la doctrine officielle - avait déploré l'absence de tribunaux militaires et les peines légères encourues par les 
militaires en cas de refus d'obéissance alors qu'à l'étranger ce crime commis devant l'ennemi était puni de la peine capitale. La rumeur s'empara de ce propos pour dénoncer un ministre prêt à condamner à mort de futurs conscrits, alors même que le ministre, sur son blog en 2010, s'était déclaré hostile à la conscription, non par principe, mais pour des raisons techniques ${ }^{1}$. Le spectre du « billet rouge » (akagami赤紙) - la couleur du document envoyé naguère aux conscrits notifiant l'incorporation sous les drapeaux - va-t-il donc resurgir au Japon ? Depuis le retour au pouvoir en décembre 2012 de Abe Shinzō 安倍晋三, président du Parti libéral-démocrate (PLD), l'agenda politique japonais a été dominé par l'adoption d'un paquet de mesures législatives controversées touchant la protection des secrets d'État d'une part (tokutei himitsu no hogo ni kansuru hō 特定秘密の 保護に関する法 2013), la sécurité nationale d'autre part (anzenhoshō kanren hō 安全保障関連法 2015) : ces dernières en particulier lèvent les restrictions constitutionnelles traditionnelles concernant l'exercice - partiel - du droit d'autodéfense collective (shüdanteki jieiken 集団的自衛権) et facilitent le déploiement des Forces d'autodéfense à l'étranger dans le cadre d'opérations de sécurisation internationale dépassant le seul cadre onusien. Ces dispositifs s'inscrivent dans une politique de réaffirmation et de globalisation, au printemps 2015, du partenariat stratégique avec Washington afin de faire face à des menaces et tensions multiformes, mais aussi de montée en puissance et de diversification des missions des FAD inaugurée depuis le début des années 1990 avec la participation des FAD aux opérations de maintien de la paix de l'ONU. Ils ont suscité de nombreuses discussions, à l'intérieur comme à l'extérieur, sur leur impact sur le « pacifisme constitutionnel » et, plus largement, sur la transformation du Japon d'un pays « promoteur de paix » en un pays « capable de faire la guerre ». Avec des formes de contre-mobilisation de la société civile japonaise inédites depuis les manifestations contre le traité de sécurité nippo-américain de 1959-1960, au sein desquelles les collectifs du SEALDs et de l'association Kyūjō no kai九条の 会 $^{2}$, les innombrables 《cafés constitutionnels », les mères de famille - voire les

1. La rumeur est partie en fait d'un article du Tōkyō shinbun東京新聞du 16 juillet 2013 rapportant les « attaques frontales [d'Ishiba] contre la Constitution pacifique ». Voir aussi figure 1 .

2. Students Emergency Action for Liberal Democracy'. Ce collectif s'est constitué au début 2014 dans la foulée des luttes contre la législation sur les secrets d'État ; Association 
revues féminines telles que VERY - jouèrent un rôle moteur. Et un usage intensif des réseaux sociaux qui contribua à une polarisation souvent excessive des débats ${ }^{3}$.

Parallèlement, le Parti libéral-démocrate au pouvoir milite, de longue date, en faveur de la transformation des FAD en une Armée à part entière, de la reconnaissance constitutionnelle de cette Armée et de l'inscription dans la loi fondamentale de dispositions relatives à l'obligation ardente de défense nationale. La conjonction de la reconnaissance de l'autodéfense collective et de la conversion éventuelle des FAD en une Armée nationale, Kokubōgun 国防軍a relancé, dans une partie de l'opinion, la crainte d'une réintroduction de la conscription. Pire, si l'on en croit les sondages, $25 \%$ des 17-19 ans seraient persuadés que les lois de sécurité nationale contiennent des dispositions autorisant le retour à la conscription ${ }^{4}$. L'affirmation a beau être inexacte, il est vrai que sur les réseaux sociaux, et dans l'opposition parlementaire, le lien entre ces lois et la réintroduction de la conscription est souvent évoqué. La question se pose donc de déterminer les raisons pour lesquelles l'adoption de ces lois sur la sécurité nationale a relancé un débat apparemment clos depuis longtemps et quel rôle, au-delà de la crédibilité même de cette option, la référence à la conscription joue dans le débat actuel sur la défense nationale au Japon.

de l'article 9 [de la Constitution], née en 2004 sous l'égide d'intellectuels tels que Ōe Kenzaburō大江 健三郎 (1935-), Tsurumi Shunsuke 鶴見 俊輔 (1922-2015), Okudaïra Yasuhiro奥平康弘 (1929-2015) et Katō Shūichi 加藤周一 (1919-2008). La version lycéenne de ce mouvement est T-ns SOWL（ティーンズ・ソウル）. « Ne plus envoyer nos enfants et notre jeunesse à la guerre » : le vieux slogan pacifiste des années 1950 au moment de la guerre de Corée a refait surface dans le discours politique et médiatique, principalement dans le nord de l'archipel, où stationne le $5^{\text {ème }}$ régiment de la $9^{\text {ème }}$ division d'infanterie souvent sollicité dans les missions à l'étranger des FAD.

3. Se rapporter au tweet ravageur du député PLD Mutō Takaya 武藤貴也, en date du 30 juillet 2015 fustigeant « tous ces jeunes qui ne veulent pas aller à la guerre et font preuve d'un égotisme et d'un égoïsme effrénés : le résultat d'une éducation d'après-guerre cultivant l'égoïsme et l'individualisme ». Sommé de se dédire, le jeune député de 36 ans préféra quitter le parti, avant d'être rattrapé par un scandale financier et... sexuel.

4. Kyōto shinbun 京都新聞, 17 juillet 2015, d'après une enquête effectuée sur les campus des universités de Kyōto et de Shiga. 


\section{CIPANGO \\ 280 Cahiers d'études japonaises nº 24}

\section{Les FAD : une image de marque ambigüe}

Historiquement, le Japon impérial avait adopté en 1873, dans le cadre de la construction d'un État-Nation moderne, un système militaire fondé sur la conscription. La jeunesse japonaise se battit en conséquence sur tous les fronts des combats menés par le Japon impérial jusqu’à la guerre du Pacifique. La défaite de 1945 avait entrainé, outre la démobilisation des forces armées et leur rapatriement, la dissolution des institutions militaires du Japon impérial et donc la fin du service militaire ${ }^{5}$. Lors de l'élaboration de la Constitution et de la mise en place de ce qui deviendra, avec l'article 9, le pacifisme constitutionnel, le sort du service militaire était en apparence définitivement scellé ${ }^{6}$. L'abolition de la conscription ne devait pas être remise en cause avec la création des FAD en 1954, au lendemain de la guerre de Corée : à l'époque, le gouvernement japonais avait opté pour une armée de métier, dont la légitimité constitutionnelle avait été aussitôt contestée, notamment par les partis socialiste et communiste, une partie des intellectuels et des médias. Le retour à la conscription avait été à l'époque impensable, même en pleine période réactionnaire du « retour en arrière », Gyaku kōsu逆コース, qui marque la seconde moitié des années 1950 : les hécatombes de la guerre du Pacifique étaient encore présentes dans les esprits; le pacifisme, tant dans ses dimensions juridique que politique et psychologique, teinté d'un fort sentiment antimilitariste, incitait par ailleurs les gouvernements japonais à la prudence dans leur politique de remilitarisation, même partielle, de l'archipel ${ }^{7}$.

5. La loi sur le service militaire a été abrogée en novembre 1945.

6. « L’empereur est fermement déterminé à faire en sorte que le peuple ne soit plus à nouveau mobilisé dans des guerres étrangères. Quel que soit le gouvernement, quelles que soient les circonstances, la pierre angulaire de la nouvelle loi fondamentale doit prévoir des dispositions garantissant le droit des citoyens à refuser le service armé, et la non-affectation des ressources de l'État à des fins militaires »: lettre de Shiratori Toshio 白鳥敏夫 (1887-1949) au ministre des Affaires étrangères Yoshida Shigeru 吉田茂 (1878-1967) en date du 10 décembre 1945. Shiratori, ancien ambassadeur à Rome, était alors incarcéré à la prison de Sugamo巣鴨拘置所 en tant que suspect de crimes de guerre de rang A.

7. Les sondages d'opinion de la première moitié des années 1950 à la veille de la fin de l'Occupation américaine montrent que si une majorité de l'opinion publique japonaise est favorable au principe de la remilitarisation de l'archipel, elle est hostile à la réintroduction de la conscription : 49 \% selon le Yomiuri shinbun 読売新聞du 26 mars 1951, 69,6\%, 
Dans cette perspective, lors des pourparlers Ikeda-Robertson d'octobre 1953, la partie japonaise fit valoir les contraintes de tous ordres - juridiques, politiques, sociales et budgétaires - qui s'opposaient à un réarmement massif de l'archipel, notamment à la proposition américaine de constituer une armée de plus de 300000 hommes qui ne pouvait être mise en œuvre sans la réintroduction de la conscription : une option totalement inenvisageable à l'époque ${ }^{8}$. Cependant, même après 1945, la question de la conscription n'avait jamais été complètement évacuée9. Dès la création de la Réserve de police, Keisatsu yobitai 警察予備隊, de 75000 hommes, en août 1950, des voix s'étaient levées pour indiquer que la professionnalisation avait un coût, et que son budget aurait permis de lever une armée de conscription de plusieurs centaines de milliers d'hommes. À la veille même de la création des FAD en 1954, l'hypothèse de la restauration de la conscription fut évoquée à la Diète, si les effectifs recrutés sur la base du volontariat s'avéraient insuffisants, ce qui signifiait que le choix en faveur de la professionnalisation des armées n'était pas définitif et aurait pu être réévalué en fonction de l'évolution des

d'après le Mainichi shinbun 毎日新聞du 14 avril 1952. Les organisations étudiantes autour de la Zengakuren 全学連et de la Wadatsumikaiわだつ夕会 - association d'intellectuels et de familles d'étudiants morts à la guerre - militent alors fortement contre la conscription par des campagnes de pétition ayant obtenu plus de 250000 signatures à la fin 1953. Ce n'est pas non plus un hasard, si le programme de lutte du syndicat de gauche d'enseignants, le Nikkyōso日教組, pour 1953 mentionne explicitement le refus de la conscription : rapport de la Commission consultative centrale sur l'éducation concernant les activités politiques des enseignants, 18 janvier 1954 : http://www.mext. go.jp/b_menu/shingi/chuuou/toushin/540101.htm. Consulté le 4 avril 2017.

8. Asabishinbun朝日新聞, 25 octobre 1978. Dès janvier 1952, le chef d'état-major du Far East Command, le général Doyle O. Hickey (1892-1961) avait indiqué à Tatsuya Eiichi 辰巳 栄一 (1895-1988), le conseiller de Yoshida Shigeru pour les affaires militaires que, pour faciliter le retrait des forces américaines et assurer sa propre défense, le Japon devait se doter d'une force de 325000 hommes : Nishikawa, 2008, p. 68.

9. Pour un plaidoyer en faveur de la conscription en tant qu' « ossature de la jeunesse nationale », Kitazawa Naokichi 北沢 直吉 (1901-1981), parti démocrate-libéral (minshu jiyūtōe主自由党), chambre des Représentants shügiin衆議院, commission du budget, 13 février 1950. Sur l'écho à la Diète du lien entre les accords de sécurité nippo-américain, le réarmement et le retour éventuel à la conscription, intervention de la Conseillère PLD, Yamamoto Sugi 山本杉 (1902-1995), chambre des Conseillers参議 院, commission sur les accords de sécurité nippo-américains, 12 juin 1960. 
282 Cahiers d'études japonaises nº 24

recrutements ${ }^{10}$. Mais le contexte politique et psychologique étant particulièrement défavorable, la professionnalisation de ces nouvelles forces de sécurité ne fut pas remise en cause. D'ailleurs la gauche japonaise ne s'était pas privée, à l'époque, de critiquer l'hypocrisie du profil bas observé par le Cabinet Yoshida en matière de remilitarisation au motif que la création de la Réserve de police constituait la première étape d'un processus conduisant, à terme, à la restauration du service militaire, que les effectifs recrutés étaient insuffisants pour satisfaire aux besoins gouvernementaux, et que de nombreuses collectivités locales votaient des mesures de sécurité publique pour étouffer l'opposition populaire, et de la jeunesse en particulier, contre la conscription ${ }^{11}$. En fait, l'opposition au service militaire couvrait alors un large spectre politique, au-delà de la ligne de démarcation entre majorité et opposition à la Diète.

La conséquence de ce choix en faveur d'une armée de métier, et dans le contexte particulier de la création des FAD, fut de couper le cordon ombilical entre la nation et les « forces armées », auxquelles les gouvernements conservateurs successifs déniaient d'ailleurs cette qualité, et de provoquer une fracture politique durable dans le Japon d'après-guerre, qui ne s'est pas totalement réduite. Du même coup, la condition militaire dans le Japon d'après-guerre fut fortement dévalorisée car, à la différence des Armées impériales, les FAD n'étaient pas un facteur - relatif de promotion sociale ; les valeurs de l'après-guerre se voulaient aux antipodes du militarisme et des vertus martiales du Japon impérial ; les FAD passaient, à tort ou à raison, pour un lieu de récupération des jeunes en mal d'intégration sociale, surtout ceux issus des 《quartiers de ségrégation » (Burakumin部落民) et les militaires avaient parfois du mal à se marier. Les syndicats d'enseignants, souvent

10. Kimura Tokutarō木村 篤太郎 (1886-1882), directeur de l'agence de Sécurité (bōanchōo 保安庁), chambre des Conseillers, commission du Cabinet, 20 mai 1954.

11. Kawakami Kan.ichi 川上貫一 (1888-1968), PCJ, chambre des Représentants, séance plénière, 27 janvier 1951; dans le même sens, Nashiki Sakujirō 梨木 作次郎 (1907-1993), PCJ, chambre des Représentants, séance plénière, 25 janvier 1952 ; sur le démenti du gouvernement : Ōhashi Takeo 大橋武夫 (1904-1981), ministre d'État en charge de la Réserve de police, chambre de Représentants, commission du budget, 23 février 1952, Yoshida Shigeru, Premier ministre, chambre des Conseillers, commission du budget, 6 mars 1952. Yoshida s'opposait ainsi, dans le camp conservateur, à Hatoyama Ichirō 鳩山一郎 (1883-1959), ouvertement favorable à la révision de la loi fondamentale, au réarmement et à la conscription : NAKAJIMA, 2016, p. 110. 
classés à gauche, militaient contre les programmes d'éducation civique jugés incompatibles avec le pacifisme constitutionnel, les campagnes de recrutement des FAD, ou de «sensibilisation à l'emploi » (shokuba taiken 職場体験), menées auprès des lycéens, voire des collégiens ; certaines collectivités locales refusaient aux antennes régionales des FAD l'accès aux registres d'immatriculation locale à des fins de recrutement ${ }^{12}$; en outre, il n'était pas rare que les enfants des militaires fussent ostracisés dans les écoles et universités japonaises. Bref, les FAD avaient mauvaise presse ; leur image était ternie par les dissensions politiques sur les questions de défense, le souvenir honni des armées impériales, voire les « rumeurs complotistes » attribuant aux FAD des velléités de subversion de l'ordre constitutionnel; elles étaient considérées comme des supplétifs des forces américaines, « voleuses d'impôt », (Zeikin dorobón税金泥棒). Il faudra des années - quinze ans - pour que les pouvoirs publics entreprennent de mesurer régulièrement le pouls des FAD auprès de l'opinion. En première ligne dans la lutte contre les catastrophes naturelles nationales, puis internationales, les FAD, par une habile politique de communication, ont su peaufiner l'image d'une force d'intervention au service de la communauté nationale et des citoyens, mais en occultant leur mission principale : la défense nationale. À partir des années 1990, le déploiement des FAD sur des théâtres extérieurs dans le cadre, notamment, des opérations de maintien de la paix de l'ONU vues au départ avec méfiance par l'opinion, a contribué également à redorer le blason de la Jieitai auprès du public, en tant que « force de paix ». Enfin, la conjoncture économique défavorable, les

12. Pour un exemple récent de refus de collaboration, celui de la municipalité de Fukuyama (département de Hiroshima), Mainichi shinbun, 24 août 2015. Ces refus tendent cependant à se raréfier : 199 cas en 2010, contre 12 seulement en 2015. Ce droit de consultation est bien prévu par l'article 120 de l'ordonnance du Cabinet d'application de la loi sur les FAD no 179 du 30 juin 1954 mais n'impose pas d'obligation aux maires et gouverneurs. C'est à l'automne 1966 qu'un journaliste de l'agence de presse Kyōdō, (kyōdō tsūshinsha共同通信社), révéla que les collectivités locales, à l'instigation de l'agence de Défense, fournissait des listes de jeunes de 16 à 25 ans pour des campagnes de recrutement. La gauche estudiantine et l'opposition s'étaient aussitôt insurgées contre une pratique qui aurait constitué, selon elles, l'antichambre de la conscription. 
tensions sur le marché de l'emploi après l'éclatement de la bulle spéculative et dix ans de récession avaient rendu relativement plus attractif le métier militaire ${ }^{13}$.

Les recompositions politiques successives depuis 1993, la reconnaissance de la constitutionnalité des FAD par le Parti socialiste japonais (Nihon shakaitō日 本社会党), ainsi que l'avènement de gouvernements de coalition ayant eu pour effet de marginaliser les forces politiques les plus hostiles aux FAD, les questions théologiques sur la constitutionnalité des FAD sont passées au second plan. Pour autant, en dépit d'une image de marque positive qui ferait pâlir d'envie une classe politique souvent décriée, les FAD ont eu également à se plaindre, moins d'une opinion publique hostile que des « politiques » : méconnaissance des dossiers ${ }^{14}$; indifférence à un secteur de l'action publique qui engrange peu de voix aux élections, « contrôle civil »- prédominance des civils sur les militaires dans le processus de décision - jugé tatillon, vexatoire et contreproductif en cas de circonstances exceptionnelles; marginalisation des militaires dans les distinctions honorifiques. Les réformes de l'appareil militaire - érection de l'agence de défense en ministère de plein exercice, réorganisation des structures d'état-major et du renseignement, assouplissement du contrôle civil - répondent sans doute à quelques-unes de ces frustrations. Mais elles suscitent en retour de nouvelles inquiétudes sur l'évolution des rapports de force entre civils et militaires dans le Japon d'après-guerre.

\section{La « vulnérabilisation » des FAD}

Contrairement à la plupart de ses alliés, mais aussi de ses adversaires, réels ou potentiels, le Japon d'après 1945 n'a jamais eu à faire la guerre ni été entraîné dans un conflit impliquant l'usage de la force armée. Un état de fait qui devait

13. Selon les sondages gouvernementaux annuels sur les questions de défense, $68,8 \%$ des personnes interrogées avaient une opinion favorable des FAD en 1969, et 92,2 \% en 2015 : Sankei nyūsu産経ニュース, 27 mars 2015.

14. D'où la proposition iconoclaste de l'ancien chef d'état-major des Forces aériennes d'autodéfense, le général Tamogami Toshio田母神 俊雄, d'obliger les hauts fonctionnaires et les candidats aux élections nationales à faire un stage de six mois dans la Jieitai : Hyōgensha 表現者, novembre 2010, nº 33, p. 22 et s. Le général Tamogami, qui s'était distingué par des positions historiques ouvertement révisionnistes et contraires aux positions officielles du gouvernement, avait été limogé en octobre 2008. 
peut-être moins à l'existence même des FAD et au pacifisme constitutionnel qu'au caractère dissuasif des accords de sécurité nippo-américain. Les tiraillements politiques sur les questions de défense ont par ailleurs longtemps entravé la mise en place de dispositifs de gestion de crise et de situations d'exception. En d'autres termes, les FAD étaient une « armée » virtuelle : elle n'avait jamais été engagée sur un champ de bataille, ni subi véritablement l'épreuve du feu. D'autant plus que les politiques avaient pris un soin extrême d'éviter de trop les exposer dans des situations comportant un risque militaire. C'était là indiscutablement l'une des raisons de la «popularité » grandissante des FAD : elles accomplissaient leurs missions essentiellement humanitaires sans avoir eu pratiquement à tirer un seul coup de feu. Or les lois sur la sécurité nationale qui sont entrées en vigueur en avril 2016 viennent en quelque sorte remettre en cause le consensus mou autour des FAD : non seulement les FAD ont vocation à assurer la défense du pays contre toute agression car, en dépit de l'hypothèse jugée désormais obsolète d'une invasion massive du territoire national par des forces étrangères, les conflits récurrents de souveraineté territoriale et les menaces susceptibles de peser sur les «zones limitrophes » de l'archipel dont le législateur japonais s'était déjà préoccupé en 1999, sont porteurs d'un risque militaire non négligeable. Mais en plus, l'autodéfense collective et la participation accrue du Japon à des actions de sécurisation internationale - en dépit là aussi des précautions d'usage quant au déploiement des FAD et à la nature de leurs missions - accroissent sensiblement leur exposition au risque. C'est ainsi que le débat autour de ces lois de sécurité nationale s'est focalisé sur deux questions : l'engrenage, c'est-à-dire la possibilité pour le Japon de se retrouver, au nom de la crédibilité de l'autodéfense collective, engagé dans un conflit dans lequel il ne serait pas initialement directement impliqué ; l'exposition au feu ennemi dans ses deux acceptions tout autant traumatisantes : celui de donner la mort ou d'être tué en situation de combat. On comprend que, dans la situation précédemment décrite, c'est ce dernier point qui est chargé d'une connotation affective et émotionnelle particulière : il ne s'agit plus en effet de parler des FAD comme d'une entité abstraite et collective ayant l'usage de la force armée pour outil, mais plus concrètement des membres des FAD, pris individuellement ou collectivement, les Jieikan 自衛官, appelés à risquer leur vie, et celle des autres, non plus seulement pour la défense du territoire national, mais au nom de la solidarité internationale et du maintien de la paix. 


\section{CIPANGO \\ 286 Cahiers d'études japonaises nº 24}

En 2015, à la question de savoir si « elles étaient d'accord pour avoir un proche dans la Jieitai », 70,4\% des personnes interrogées ont répondu positivement, soit un recul de 2,1\% par rapport par rapport à 2012. Et pour 75,1\% de celles qui ont répondu négativement, « c'est un travail exposé en cas de guerre », un pourcentage en hausse là aussi de 3,8\%. L'impact des discussions autour des lois sur la sécurité nationale est ici indéniable. Ce n'est pas un hasard non plus si l'on observe un regain d'intérêt pour les stratégies d'évitement du service militaire actuellement développées par les jeunes sud-coréens, et les appelés américains durant la guerre du Vietnam, le restauration du service militaire en Lituanie et en Ukraine, en Suède où le service militaire supprimé en 2010 a été réintroduit en mars 2017, voire pour le système de recensement, le Selective Service System, en vigueur aux États-Unis, obligatoire pour tous les jeunes hommes âgés de 18 à 25 ans. Il s'agit de faire valoir que l'abolition présumée du service militaire n'est en réalité qu'une «suspension », et qu'il est toujours possible de revenir en arrière, pour pallier les difficultés de recrutement d'une armée de métier dans un contexte de tensions aux frontières.

\section{Autodéfense collective et conscription}

C'est dans ce contexte particulier que les rumeurs de réactivation de la conscription ont refait surface, notamment sur les réseaux sociaux, sur la base d'un discours dont les prémisses sont complémentaires : le service militaire pourrait être à terme rétabli car, du fait de l'autodéfense collective et des engagements croissants des FAD à l'international, les effectifs d'une armée de métier seraient insuffisants pour faire face à ces nouvelles missions. Ou bien, du fait de l'aversion au risque, de nombreux militaires pourraient démissionner et il y aurait de moins en moins de volontaires pour intégrer la Jieitai. D'après plusieurs personnalités politiques, l'effet de ciseau induit ne manquerait pas d'occasionner une baisse des effectifs et de la qualité de recrutement des FAD ${ }^{15}$. En d'autres termes, le service militaire

15. Nonaka Hiromu 野中 廣務, ancien secrétaire général (kanjichō幹事長) du PLD, cité dans Asahi shinbun, 23 mai 2014; dans le même sens, Katō Kōichi加藤 紘一, également ancien secrétaire général du PLD, Akahata shinbun 赤旗新聞, 18 mai 2014; Koike Kiyohiko小池 清彦, maire de Kamo 加茂市 (département de Niigata), Asahi shinbun, 25 juin 2014. Koike est un ancien cadre de l'agence de défense (Bōeichō 防衛 
viendrait suppléer un mouvement de désaffection à l'égard des FAD, tant en ce qui concerne les effectifs actuellement sous les drapeaux que les réserves. Ces rumeurs sont alimentées par des extrapolations à partir d'initiatives locales fournissant aux FAD la liste des élèves de $3^{\text {ème }}$ année de lycée ${ }^{16}$, la hausse des départs enregistrés en $2014^{17}$, ou par des programmes de stages conclus entre certaines entreprises et le ministère de la Défense en juillet 2013 aux termes desquels les jeunes salariés recrutés pourraient faire une immersion d'un à deux ans dans les FAD, qu'ils ne pourraient refuser puisqu'il s'agirait d'une obligation de service. Du côté du ministère de la Défense, il s'agirait d'anticiper une baisse éventuelle des effectifs et de renforcer les forces de réserve des FAD très insuffisantes - 32000 hommes - en 2014, soit $70 \%$ seulement des effectifs légaux du corps de réserve ${ }^{18}$. Il existe déjà

庁) qui s'était opposé à l'envoi des FAD en Irak ; cf. la conférence de presse du député Murakami Seiichirō 村上 誠一郎du 27 juin 2014, également critique à l'égard de la position du Cabinet Abe. Il est vrai cependant que, selon la direction des statistiques du ministère des Affaires générales (sōmushōo総務省), le vivier de recrutement des FAD - la population des jeunes hommes âgés de 18 à 26 ans - tend à diminuer : 8,92 millions en 1994, contre 5,69 millions seulement en 2012, soit une diminution d'environ $36 \%$ : d'après le Portail officiel de la Statistique (http://www.e-stat.go.jp/SG1/estat/ List.do ?bid=000001039703\&cycode $=0$ ). Le nombre de postulants tend également à diminuer par rapport à 2013: 7334 candidats en 2015 aux postes d'encadrement ouverts aux diplômés de l'enseignement supérieur contre 9077, soit une diminution d'environ $20 \%$; De façon générale, le nombre de postulants aux FAD (carrière) qui avait atteint un pic de 51192 en 2011 n'était plus que de 31145 en 2015. La baisse est également sensible pour les candidats à l'engagement : de 33534 candidatures en 2013 à 31361 en 2014. Officiellement, on attribue cette baisse à une conjoncture économique plus favorable. Toutefois, selon un document interne de l'état-major de la région Est en date de décembre 2014 : « la poursuite de la situation démographique actuelle rend hors d'atteinte à terme les objectifs de recrutement des FAD » et en avril 2015, les directeurs des bureaux régionaux de recrutement de Kyūshū九州 et d'Okinawa 沖縄reconnaissaient également « l'impact des informations relatives à l'autodéfense collective », ainsi que la nécessité d'un effort d'explication en direction des familles inquiètes : FUSE, 2015, p. 22.

16. Kōbe shinbun 神戸新聞, 20 octobre 2015.

17. 12500 départs en 2014 contre 11939 en 2013.

18. Ce « programme » a été divulgué à la chambre des Conseillers par Tatsuya Kōta辰巳 孝太, parlementaire du Parti communiste japonais à la fin août 2015 et aurait été mis au point à l'initiative de la centrale patronale Keizai dōyükai 経済同友会, Nikkan gendai 日 刊ゲンダイ,29 août 2015. 
des stages dans les FAD organisés à destination des nouveaux salariés, notamment dans les sociétés qui entretiennent des liens commerciaux avec la Jieitai ${ }^{19}$. En outre, la communication des FAD est plus agressive tant en direction des familles - les visites à domicile et autres contacts directs avec l'entourage familial se sont intensifiés - que des lycées reconnus comme pourvoyeurs de soldats, avecle concours des anciens élèves ayant intégré la Jieitai. En juillet 2014 enfin, le ministère de la Défense, en plein débat autour de la nouvelle interprétation concernant le droit à l'autodéfense collective, et aux lendemains du $60^{\text {ème }}$ anniversaire de la création de la Jieitai, a lancé une grande campagne de recrutement au profit des FAD en mobilisant le groupe pop AKB 48 très en vogue auprès d'une partie la jeunesse ${ }^{20}$. Naturellement l'opposition n'allait pas tarder à s'emparer de la question : en juin 2015, la commission d'information du Parti démocrate avait fait parvenir à ses antennes locales un poster ${ }^{21}$ avec pour titre « $\grave{A}$ quand la conscription ? Une inquiétude qui monte », à destination des mères de famille, représentant un personnage vêtu d'un uniforme militaire aux côtés d'une figure féminine inquiète arborant une oriflamme sur laquelle est inscrite la mention « incorporation ». Le titre chapeaute un court texte critiquant le changement d'interprétation constitutionnelle concernant l'autodéfense collective : un revirement qui pourrait également s'appliquer à la conscription. L'ensemble évoque, sous une forme détournée, le départ sur le front. Le personnage masculin, à l'allure juvénile, n'arbore pas un uniforme des FAD, mais celui de l'ancienne armée impériale de terre : il s'agit d'associer le retour éventuel de la conscription à la guerre qui s'attache aux armées impériales. Le trait du dessin est léger, proche du manga. On perçoit bien que le parti cherche ainsi à accrocher la jeunesse. Mais le dessin dénonce aussi, de façon subliminale, la « légèreté » avec laquelle le gouvernement Abe entend exposer désormais la vie des soldats par sa politique de défense. Sur les réseaux sociaux, le secrétaire général du Parti communiste japonais (PCJ, Nihon kyōsantō日本共産党), Shii Kazuo志位和夫, a pris une position semblable. Par

19. Akahata shinbun, 16 septembre 2015, à propos de la firme NTT. Selon le ministère de la Défense (Bōeishō防衛省), ce sont ainsi 13401 nouveaux salariés qui auraient bénéficié de ces stages en 2014, en grande majorité dans les Forces terrestres d'autodéfense.

20. Tōkyō shinbun, 6 août 2014.

21. Voir figure 2. 
ailleurs, pour l'opposition, l'exemple de l'autodéfense collective constitue un précédent : après avoir soutenu pendant des décennies que le Japon sur le plan du droit international était titulaire d'un tel droit, mais ne pouvait l'exercer en raison de l'article 9 de sa Constitution, le gouvernement japonais a décidé de modifier en juillet 2014 cette interprétation, ce qui a été le prélude au paquet législatif sur la sécurité nationale de l'été 2015. En d'autres termes, fort de ce « précédent », rien n’empêcherait le gouvernement japonais de modifier, le cas échéant, sa position sur l'inconstitutionnalité de la conscription : telle est du moins le point de vue exprimé par le secrétaire général du Parti démocrate, Edano Yukio枝野幸男, et le président du parti Okada Tatsuya 岡田克也, en juin et novembre $2015^{22}$. Le 21 juin, après l'adoption des lois sur la sécurité nationale par la chambre des Représentants, le président de la Commission des études politiques du Minshutō (Minshutō seisaku chōsa kaichōe主党政策調查会長), Hosono Gōshi細野 豪志, indiquait sur son blog que, pour des raisons démographiques - la diminution de la population japonaise -, et du fait de l'élargissement des activités des FAD, « dans la génération de mes petits-enfants, une personne sur dix intégrera la Jieitai $\gg$. Un autre parlementaire du parti avait indiqué peu de temps auparavant publiquement à la Diète que « ce qui inquiète le plus mon épouse, c'est que notre fils aîné, âgé de 1 an, soit à l'avenir soumis à la conscription ${ }^{23} \gg$. Ce tir de barrage ne faisait pas toutefois l'unanimité à l'intérieur du Minshutō : certains déploraient une fuite en avant du Parti, un aveu de faiblesse, et un risque d'atteinte au moral des FAD. Au début juillet, le poster précité avait été jugé « inapproprié » par le 《 ministre » des Affaires étrangères du «Cabinet fantôme » (jiki naikaku次期 内閣), Nagashima Akihisa長島昭久, qui avait annoncé des 《 rectifications 》

22. Dans le même sens, les positions de Sakata Masahiro阪田雅裕, ancien directeur du bureau législatif du Cabinet : outre le fait que changer l'interprétation de la loi fondamentale est plus facile à réaliser que la révision de la loi fondamentale, Sakata souligne que la consolidation de l'interprétation gouvernementale sur la prohibition constitutionnelle de la conscription était plus fragile que pour le droit d'autodéfense collective. Elle est donc aujourd'hui plus exposée par la nouvelle doctrine du Cabinet Abe : Akahata shinbun, 12 juillet 2014.

23. Terada Manabu 寺田 学, chambre des Représentants, commission spéciale relative à la législation sur la paix et la sécurité nationale et internationale, 19 juin 2015. 


\section{CIPANGO \\ 290 Cahiers d'études japonaises nº 24}

adéquates $^{24}$ : les 500000 exemplaires du poster litigieux furent mis au pilon et de nouveaux posters mis en circulation à la fin du mois. S'ils rappellent la fragilité de la position gouvernementale sur la conscription, ils ne renferment plus d'allusions plus ou moins explicites aux anciennes armées impériales.

\section{Les effets pervers de l'abaissement de la majorité électorale ?}

Il n'est pas jusqu'à une réforme a priori positive - le droit de vote accordé depuis juin 2015 aux jeunes de 18 ans à la suite de la modification de la loi sur les fonctions publiques électives - qui ne suscite la suspicion dans les réseaux sociaux. D'autant plus qu'elle intervenait dans un climat politique tendu du fait des discussions autour des nouvelles lois sur la sécurité nationale. Si cette réforme aligne le Japon sur les pratiques en vigueur dans 176 pays, elle n'était pas une revendication majeure de la société civile ou de la jeunesse japonaise, et l'opinion publique reste d'ailleurs partagée sur la question. Il fallait donc, pour certains commentateurs, trouver d'autres raisons que le souci d'encourager la participation politique des jeunes avancé par la classe politique nippone alors que, traditionnellement, le taux de vote des électeurs les plus jeunes est faible ${ }^{25}$. Sont concernés au premier chef par cette réforme les jeunes de 18 ans, ceux qui sont en dernière année de lycée. Le corps électoral va donc être mathématiquement augmenté de 2.400 .000 électeurs potentiels. Mais il se trouve aussi que les jeunes de 18 ans constituent le cœur de cible du recrutement des FAD. Là aussi, le chiffon rouge de la conscription a été agité : à partir du moment où les jeunes accèdent à la responsabilité politique, ils devraient aussi en assumer les contreparties. On fait aussi observer que, dans la plupart des pays concernés, l'abaissement de la majorité électorale allait de pair avec le service militaire. Certes, ce constat ne signifie pas à lui seul que le Japon devrait s'engager sur la voie de la réinstallation de la conscription, mais l'abaissement de la majorité électorale lèverait un obstacle démographique à l'enrôlement éventuel

24. Sankei nyūsu, 25 juin 2015 ; ibid., 5 juillet 2015. Les divergences d'appréciation sur ce sujet controversé sont anciennes : en septembre 1999, en compétition pour la présidence du Parti, Hatoyama Yukio鳩山由紀夫 n’avait pas exclu la réintroduction de la conscription en cas de circonstances exceptionnelles, contrairement à ses deux autres challengers : agence de presse Kyōdō, 14 septembre 1999.

25. Nikkan Gendai, 7 juillet 2015. 
de futurs conscrits. En d'autres termes, alors qu'aux États-Unis l'abaissement de la majorité électorale avait été conçue pour mettre un terme à la discrimination dont les jeunes étaient victimes, puisqu' ils pouvaient être appelés sous les drapeaux tout en étant privés de leurs droits politiques, au Japon, l'octroi des droits politiques aux jeunes serait le préalable à la conscription. C'est du moins ce qui domine nettement sur les réseaux sociaux, et au PCJ, pour lesquels l'exemple américain illustre bien cette liaison intrinsèque entre l'abaissement de la majorité électorale et la conscription ${ }^{26}$. Bien plus, selon certains hebdomadaires, on soupçonne le gouvernement de double jeu : séduire la jeunesse par l'abaissement du droit de vote dans l'espoir de provoquer, en retour, un réflexe d'adhésion en faveur du PLD et d'une hypothétique révision de la loi fondamentale, tout en occultant la liaison de fait entre le droit de vote et la conscription, naturellement admis par certains parlementaires du PLD ${ }^{27}$. Certes il n'a pas échappé à la classe politique dans son ensemble que le hasard du calendrier parlementaire - mais en était-ce vraiment un ? - faisait pratiquement coïncider la promulgation de cette loi avec l'adoption par la chambre de Représentants du paquet législatif sur la sécurité nationale. En tout cas, l'initiative du Cabinet a embarrassé ce qui reste de la gauche japonaise à la Diète : le Parti social-démocrate et le Parti communiste ne sont pas associés à la proposition de loi d'initiative parlementaire présentée par les autres formations, compte tenu des circonstances politiques l'entourant et des arrière-pensées supposées du gouvernement, mais ils l'ont tout de même votée. Préconisée de longue date par l'opposition, l'opinion n'aurait pas compris que la fixation de la majorité électorale à 18 ans soit prise en otage par les controverses sur la sécurité nationale. Mais l'abaissement de la majorité électorale n'est pas exempt d'effets secondaires. Sur les réseaux sociaux, le lien entre l'abaissement de la majorité électorale et la conscription est beaucoup plus explicite. Outre le fait que l'abaissement de la majorité électorale va créer deux catégories de lycéens : ceux titulaires du droit de vote et ceux qui en sont encore privés, et fractionner politiquement les jeunes - un argument spécieux souvent avancé pour contester

26. Shükan zenshin週刊前進 $\mathrm{n}^{\circ} 2719 \mathrm{du} 1^{\text {er }}$ février 2016.

27. Shükan asahi週刊朝日, 29 mai 2015. Voir la position de Nishida Shôji西 田 昌司, membre de la chambre des Conseillers : https://www.youtube.com/ watch?v=SBJdoCXWLqY. Consulté le 21 août 2017. 


\section{CIPANGO}

292 Cahiers d'études japonaises n²4

la réforme - il sert également de levier au renforcement du contrôle politique des enseignants, souvent rétifs à la réorientation de la politique de défense du gouvernement Abe. Naturellement, le ministère de l'Éducation et les politiques se sont intéressés aux conséquences de l'abaissement de la majorité électorale. C'est ainsi qu'en octobre 2015, la direction de l'éducation primaire, des collèges et des lycées a rendu publiques les directives sur l'encadrement des activités politiques des lycéens dans l'enceinte ou à l'extérieur de l'école ${ }^{28}$. Le PLD quant à lui, soutenu en cela par le Sankei shinbun et le Yomiuri shinbun, soucieux d'éviter que l'école ne devienne le champ clos des joutes partisanes et d'une « éducation orientée » (henkō kyōiku 偏向教育), a mis à profit le délai entre la promulgation de la réforme de la loi sur les emplois publics électifs et son entrée en vigueur pour envisager les dispositifs propres à « sanctuariser » les lycées. En mai 2016, à la veille des élections pour le renouvellement de la chambre de Conseillers pour lesquelles ces nouveaux électeurs allaient voter pour la première fois, le PLD avait confirmé qu'il souhaitait un réaménagement de la législation sur le statut des enseignants du public et du privé et la scolarité obligatoire, afin de préserver la « neutralité politique de l'enseignement », (kyōiku no chüritsu 教育の中立), en l'assortissant de sanctions pénales ${ }^{29}$. En juin de la même année sur le site du PLD, il était même possible de télécharger un formulaire où l'on pouvait rapporter les manquements observés sur le terrain à cette obligation de neutralité susceptibles d'être transmis à la police ${ }^{30}$. Devant les protestations face à ce véritable système de délation, le PLD avait été conduit à supprimer cette page au bout d'un mois mais

28. Notification, $t s \bar{u} c h i$ 通知, $\mathrm{n}^{\circ} 933 \mathrm{du} 29$ octobre 2015 sur l'éducation politique dans les lycées et sur les activités politiques des élèves des collèges : http://www.mext.go.jp/b_ menu/hakusho/nc/1363082.htm. Consulté le 20 août 2017.

29. Ce fut l'objet de la loi $\mathrm{n}^{\circ} 157$ du 3 juin 2017 portant dispositions spéciales sur la consolidation de la neutralité politique de l'enseignement dans les établissements scolaires en charge de l'instruction obligatoire. Les contrevenants s'exposent, en vertu de l'article 4, à une peine de travaux forcés inférieure à un an ou bien à une amende qui ne peut dépasser 30000 yens : http://www.houko.com/00/01/S29/157.HTM. Consulté le 20 août 2017. Des peines sensiblement inférieures à ce que réclamait initialement le PLD : trois ans maximum de travaux forcés ou une amende inférieure à 1 million de yen.

30. http://www.huffingtonpost.jp/2016/07/09/ldp-education-investigation_n_10902 078.html. Consulté le 20 août 2017. 
sans que l'on sache si l' « enquête » à la base de ce formulaire avait été suspendue. Le Jimintō avait été contraint de reconnaître que militer pour la paix ou contre la guerre ne constituait pas la manifestation d'une « éducation orientée ». En revanche contester les projets de loi sur la sécurité nationale sur la base de cet engagement militant l'aurait été. Les plus critiques à l'égard du PLD ont évoqué la mise en place d'un système de « contrôle de la pensée », rappelant les pires heures de la loi sur la préservation de la paix publique de 1925, chian ijihō治安維持法 ${ }^{31}$. Sur un autre plan, un rapport présenté au $66^{\text {ème }}$ congrès de recherche pédagogique du syndicat Nikkyōso mettait en garde contre les nouvelles modalités des bilans de santé des élèves, en rapport avec la volonté du Premier ministre de réviser la loi fondamentale et la politique de défense du pouvoir ${ }^{32}$.

\section{Les contre-feux du gouvernement}

Face à ces rumeurs persistantes concernant le service militaire, les pouvoirs publics ont développé une stratégie à plusieurs volets : 1) le service militaire serait inutile car avec en moyenne plus de quatre candidats pour un poste, la Jieitai, dispose d'une marge de manœuvre relativement importante lui permettant de maintenir son niveau actuel de recrutement ; 2) l'ère des levées en masse est révolue : les guerres, les matériels ont considérablement évolué : ils requièrent des compétences technologiques et informatiques de plus en plus complexes et sophistiquées, dont la maîtrise requiert du temps et qui ne sont pas à la portée immédiate du premier appelé ; 3) la restauration du service militaire aurait un coût humain et financier important : outre le paiement d'une solde, il faudrait construire de nouvelles installations et casernes, affecter un personnel important à la formation des appelés : un luxe que les finances publiques japonaises ne peuvent pas se permettre et, dans le même ordre d'idée, les pouvoirs publics font valoir qu'en période de crise démographique, il serait absurde de soustraire du marché du travail, et donc de la création de richesse, une classe d'âge entière ; 4) la situation géostratégique du Japon est différente de celle de la Corée du Sud qui, elle, a maintenu la conscription en raison de la présence de plus d' 1 million de soldats nord-coréens

31. Mainichi shinbun, 28 juillet 2016. Également Kyōiku shinbun, 22 juillet 2016.

32. Daily shinchōデイリー新潮, 21 février 2017. 
massés au-delà du $38^{\text {ème }}$ parallèle ${ }^{33}$; 5) la plupart des grands pays, dont ceux du G7, ont renoncé à la conscription : c'est bien la preuve que le service militaire, dans son acception traditionnelle, ne correspond plus aux besoins et aux défis d'une armée moderne ; 6) le gouvernement considère que le service militaire est inconstitutionnel : il s'oppose à l'article 18 de la loi fondamentale qui prohibe toute « sujétion » et autres « servitudes » involontaires, ainsi qu'à son article 13 qui garantit aux citoyens le « droit à la vie », à « la poursuite du bonheur » et au « respect de la dignité individuelle ». Une position maintes fois affichée par le PLD au pouvoir et rappelée à la Diète par le Premier ministre Abe Shinzōo ${ }^{34}$. En juillet 2014, le site du secrétariat général du Cabinet devait d'ailleurs afficher une synthèse de la position du gouvernement sur la question. Mais, en même temps, les FAD lançaient une campagne d'information et de recrutement par courriel auprès de $20 \%$ environ des jeunes gens de 18 ans $^{35}$; 7) quand bien même, dès 2005 , lorsque le PLD a commencé à travailler sur la question de la révision éventuelle de la Constitution et de son article 9, le PLD a constamment démenti les « rumeurs » faisant état d'une réactivation du service militaire, au motif qu'elle ne figure dans aucun engagement public du Parti, notamment dans ses programmes électoraux, et qu'elles relevaient d'une gesticulation démagogique de l'opposition, incapable de présenter des contre-propositions crédibles aux projets de loi sur la sécurité nationale ${ }^{36}$.

\section{Ishiba, 2015, p. 168 et $s$.}

34. Chambre des conseillers, commission du budget, 15 mai 2013 ; chambre des Conseillers, séance plénière, 27 juillet 2015 ; chambre des Conseillers, commission spéciale relative à la législation sur la paix et de la sécurité nationale et internationale ; 30 juillet 2015. Également Suga Yoshihide, secrétaire général du Cabinet, en pleine manifestation contre les lois sur la sécurité nationale, Sankei shinbun, 31 août 2015. Dans le même sens, Yokobatake Yûsuke, directeur du bureau législatif du Cabinet, chambre des Représentants, commission spéciale relative à la législation sur la paix et de la sécurité nationale et internationale, 19 juin 2015, en réponse à la question orale de la députée du Parti démocrate Tsujimoto Kiyomi 辻元清美 ; Tamura, 2014, p. 186.

35. Nakatani Gen, ministre de la Défense, chambre des Conseillers, commission spéciale relative à la législation sur la paix et la sécurité nationale et internationale, 9 septembre 2015.

36. Sur les bruits relatifs à la conscription, Akahata shinbun, 28 novembre 2005, dépêche de l'agence de presse Kyōdō du 4 mars 2010, et le démenti officiel du secrétaire général 


\section{Les ambigüités persistantes}

Le problème est que ces arguments, aux dires tant des partisans que des contempteurs d'un retour à la conscription, sont loin d'être totalement irréfragables. Ainsi, pour le Parti démocrate, aucune disposition constitutionnelle, aucune décision de justice n'ayant déclaré explicitement la conscription contraire à la loi fondamentale, la position maintes fois réitérée par la direction législative du Cabinet de l'inconstitutionnalité du service militaire est une simple appréciation du pouvoir exécutif qui laisse ouverte la possibilité de la réintroduction du service militaire ${ }^{37}$. En fait, il existe toute une série de raisons qui sont avancées en faveur de la conscription.

Le niveau d'éducation de la population s'est élevé, ce qui la rendrait plus apte au maniement d'équipements plus sophistiqués que le fusil ou le pistolet; le coût d'une armée de conscription reste inférieur à celui d'une armée de métier, et tous les postes ne requièrent pas de compétences techniques supérieures : c'est déjà le cas des engagés sur contrat de deux ou quatre ans qui sont tous issus de la société civile. Au surplus, l'exemple israélien montre, si besoin est, que la conscription n'est nullement incompatible avec une armée forte et performante. Certains spécialistes des questions stratégiques tels Koizumi Yū 小泉悠, estiment que dans le cas de « guerres hybrides », qualificatif qui désigne des situations intermédiaires de conflit entre l'état de paix et l'état de guerre, le recours à la conscription pourrait être justifié. Le Japon reste entouré de pays - Russie, Chine, Taïwan, les deux Corée - qui pratiquent la conscription, et capables de ce fait de mobiliser des effectifs importants en cas de guerre et de les renouveler, alors que le vivier potentiel des FAD reste limité, et que les effectifs réels ne couvrent que $70 \%$ des effectifs budgétaires pour les hommes du rang. On a pu relever que le débat

du PLD, Ōshima Tadamori大島 理森. Sur les accusations de démagogie à l'encontre de l'opposition, interview accordée par le Premier ministre Abe, Sankei shinbun, 20 septembre 2015. Dans le même sens, éditorial du Yomiuri shinbun du 4 août 2015, accusant l'opposition de « faire des sauts dans son raisonnement » et d'alimenter des « craintes obsolètes ».

37. Terada Manabu, chambre des Représentants, commission spéciale relative à la législation sur la paix et la sécurité nationale et internationale, 15 juin 2015. 
sur le service militaire n'est pas totalement clos aux États-Unis ${ }^{38}$, que même parmi les pays qui ont supprimé ou plus exactement «suspendu » le service militaire, l'Allemagne (2011) et la France (1997), on reconnaissait à la conscription des avantages. En Allemagne, on avait prêté à l'armée de conscription le mérite d'entraver tout retour au nazisme. En France, on a mis en place une journée obligatoire de sensibilisation aux questions de défense nationale et, à gauche comme à droite, l'idée de d'une réintroduction du service militaire sous forme de mise en place d'une Garde nationale, fait son chemin ${ }^{39}$. Dans les deux cas, ce sont principalement des raisons sociopolitiques et psychologiques, plus que techniques et opérationnelles, qui ont été avancées pour maintenir ou justifier des alternatives au service militaire. Quant à « l'inconstitutionnalité » même de la conscription, elle est contestée au sein même du PLD, voire aux échelons supérieurs des FAD : ainsi pour Ishiba Shigeru, dans aucun pays au monde, la conscription est considérée comme inconstitutionnelle, sauf au Japon. Outre l'injure ainsi faite aux FAD, un pays qui considère le service militaire - donc le devoir de protéger la nation comme une « servitude » n'est pas digne d'être considéré comme un État à part entière. La thèse de l'inconstitutionnalité de la conscription est d'ailleurs critiquée par une minorité de constitutionnalistes spécialistes de questions de défense tels que Nishi Osamu西 修, Momochi Akira百地 章 ou Nagao Kazuhiro長尾一 紘 qui contestent l'assimilation de la conscription au travail forcé ou à une sorte de pratique esclavagiste ${ }^{40}$. De plus, la Cour suprême des États-Unis, en 1918,

38. En janvier 2003, juste avant la seconde guerre du Golfe, le Congrès avait été saisi d'une proposition de loi démocrate de restauration du service national pour remédier à un système de recrutement fondé sur l'inégalité sociale. Même proposition de l'ancien commandant en chef des forces américaines stationnées en Afghanistan, le général Stanley A. McChrystal, en juillet 2012.

39. Voir par exemple Le Monde, 29 novembre 2016. En mars 2017, le candidat Emmanuel Macron à la présidence de la République s'était prononcé en faveur de la restauration d'un service national d'un mois, proposition que le Sénat s'empressa de juger impraticable et d'un coût exorbitant : La Tribune, 15 juin 2017.

40. Nikkan gendai, 21 juin 2015. Ironiquement, ces trois personnalités soutenaient la thèse gouvernementale de la constitutionnalité des nouveaux projets de loi sur la sécurité nationale : Tōkyō shinbun, 11 juin 2015. Également, l'audition du professeur Nishi Osamu, chambre des Représentants, commission spéciale sur les dispositifs juridiques concernant la paix et la sécurité nationale et internationale, 22 juin 2015. 
avait rendu un arrêt selon lequel l'obligation de contribution des citoyens à la défense nationale ne constituait pas une « servitude involontaire » prohibée par la loi fondamentale. En conséquence, les fondements juridiques de la position gouvernementale demeuraient fragiles ${ }^{41}$.

L'argument selon lequel la conscription changerait l'image du Japon d'après-guerre ne tiendrait pas : la Suisse a bien rejeté par votation populaire, en septembre 2013, l'abandon du service militaire, ce qui ne l'empêche nullement d'être un État pacifique et pacifiste ${ }^{42}$... Cela ne signifie pas naturellement que le service militaire doive être nécessairement réinstallé, mais que cette décision relève d'abord de considérations politiques et stratégiques ${ }^{43}$. Bien des années auparavant, en février 1981, le général Takeda Gorō竹田五郎 avait tenu des propos pratiquement similaires, mais il avait été démis pour cela de ses fonctions de président du conseil d'état-major combiné des FAD, pour avoir critiqué la position gouvernementale comme outrageant la mission sacrée de défense nationale impartie à la Jieitai ${ }^{44}$. Enfin, lorsque le Cabinet Suzuki Zenkō (Suzuki zenkō naikaku鈴木善幸内閣), le 15 août 1980, énonce le principe de l'inconstitutionnalité de la conscription, en réponse à une question écrite d'un parlementaire, il le fait dans le cadre d'une définition précise : la « mise en place d'une armée permanente, impliquant l'appel sous les drapeaux et l'entraînement d'un contingent annuel, régulièrement renouvelé, comme organisation de base du temps de guerre » : la question de savoir si les FAD constituent une « armée permanente » étant déjà sujette à caution, les spécialistes relèvent que l'article 9, et donc le pacifisme constitutionnel, ne figure pas dans l'argumentaire

41. Maekawa Kiyoshige, Parti démocrate, chambre des Conseillers, séance plénière, 17 septembre 2015 .

42. La 《Conférence du Japon », Nihon kaigi 日本会議, brain trust de la droite nationaliste proche du Premier ministre Abe Shinzô, n'a pas pris position officiellement pour la conscription, mais l'exemple suisse est souvent cité par ses membres et ses antennes locales pour mettre en cause «le pacifisme béat », heiwaboke 平和ぼけ, de l’opinion.

43. Ishiba Shigeru, ministre en charge de la Réhabilitation régionale, chambre des Représentants, sous-commission de la commission d'enquête sur la Constitution, 23 mai 2014. Sur le risque d'assimilation de l'entrée dans les FAD à une « servitude » : TAKINO, 2014, p. 223-224.

44. Asahi shinbun, 3 et 4 février 1981. 
gouvernemental sur l'inconstitutionnalité de la conscription, alors que l'existence même de l'article 9 suffirait à clore toute discussion sur la question. Et pour cause : selon le PLD au pouvoir, l'article 9 ne fait pas obstacle à l'existence même des FAD. Et comme le gouvernement a également toujours soutenu que les Forces japonaises d'autodéfense étaient conformes au «bonheur public », traduction japonaise de l'intérêt public, il n'y aurait pas d'obstacle juridique à envisager des 《réquisitions » (Chōshū 徵集), de personnes au profit des FAD, au nom de « la protection supérieure de la liberté et des droits de la population », ce qui permettrait de contourner l'article 18 de la loi fondamentale. Autrement dit, l'interprétation gouvernementale ne serait pas suffisante pour empêcher l'appel sous les drapeaux de la jeunesse japonaise, dès lors qu'il ne prendrait pas la forme récurrente et systématique de la conscription et que d'autres articles de la loi fondamentale - les articles 12 et 13 - pourraient être réinterprétés à cette fin ${ }^{45}$. Ajoutons que quelques trente-cinq ans après cette prise de position du Cabinet Suzuki, le gouvernement Abe justifiait le recours éventuel à l'autodéfense collective dans le cadre de la protection du « bonheur public, de la liberté et des droits de la population ». Il existerait donc des justifications similaires à l'autodéfense collective et aux « réquisitions » précitées. Bien plus, le projet de révision de la Constitution rendu public par le PLD en avril 2012 contient deux dispositions problématiques : dans le préambule, il n'est plus fait mention du droit à vivre dans la paix, mais y est introduite une « obligation en matière de défense » : « le peuple japonais proclame sa volonté de protéger la nation et le pays natal avec fierté et résolution ». Ce dispositif doit être rapproché du nouvel article 9 alinéa 3 selon lequel « le peuple japonais apporte son concours au maintien de l'intégrité du territoire national ». Au sein du PLD, on s'était interrogé sur l'opportunité d'une

45. ŌE, rééd. 2013, p. 5 et 6 . On parle parfois de « conscription limitée » : si la prohibition constitutionnelle de l'article 18 n'est pas absolue, mais relative, le gouvernement pourrait ainsi réquisitionner des ingénieurs informatiques, des spécialistes des transports et communications, des personnels médicaux, des juristes, etc. Déposition de l'avocat Itō Makoto伊藤真, chambre des Conseillers, commission spéciale relative à la législation sur la paix et la sécurité nationale et internationale, 8 septembre 2015 . Le « plan des trois flèches » (mitsuya kenkyü三矢研究), élaboré dans le plus grand secret par une partie de l'état-major des FAD, et révélé à la Diète en février 1965, prévoyait également la possibilité de procéder à de telles « réquisitions », en cas de « circonstances exceptionnelles ». 
disposition plus explicite sur le devoir de défense, mais comme la concrétisation d'une telle explicitation était liée à la conscription et soulevait des problèmes de constitutionnalité, il avait été décidé de ne pas y faire référence. Toutefois, cette formulation abstraite laisserait ouverte la possibilité par le législateur de réinstaurer la conscription. D'autant plus que l'article 18 était réécrit pour prohiber certes « toute contrainte par corps pour des raisons économiques et sociales », mais non pour des raisons politiques. Pour certains analystes, le PLD souhaiterait en réalité la restauration de la conscription, mais craignant les réactions violentes de l'opinion, il s'en serait tenu à une réécriture ambiguë ménageant le statu quo, tout en laissant la porte ouverte à sa réintroduction future ${ }^{46}$. En d'autres termes, quand bien même la conscription serait contraire à la Constitution actuelle, elle ne le serait plus forcément dans le cadre d'une révision ultérieure. Quoi qu'il en soit, alors qu'au début du mois de juillet 2013, le président du QG du PLD pour la promotion de la révision de la Constitution, Hori Kōsuke保利耕輔 avait publiquement évoqué la question de la conscription parmi les « points encore en discussion », il avait été aussitôt rappelé à l'ordre par le secrétaire général du Parti, Ōshima Tadamori, pour lequel les « points » en question ne concernaient que la façon dont les États démocratiques concevaient le service militaire dans les relations entre la nation et les forces armées, et n'apportaient aucune modification à la position officielle du Parti ${ }^{47}$. Preuve néanmoins que le débat est loin d'être clos au sein même du PLD, certains responsables du PLD admettent qu'un changement d'interprétation sur l'inconstitutionnalité de la conscription reste «théoriquement » possible ${ }^{48}$. En novembre 2015, Mme Inada Tomomi 稲田 朋美, présidente de la commission de recherche politique du PLD (jimintō seimu chōsakai, 自民党政務調査会) et à ce titre l'une des figures de proue du parti conservateur, se déclarait en faveur d'une incorporation temporaire dans les FAD, à titre d'expérience, de l'ensemble des jeunes Japonais quel que soit leur sexe. Devenue ministre de la Défense, ces

46. YamaUCHI, 2015, p. 189-190; Zaikei shinbun, 5 juillet 2014.

47. Agence Kyōdō, 15 juillet 2013.

48. Funada Hajime船田 元, député PLD et président du QG sur la révision de la Constitution du PLD (jiȳu minshutō kenpō kaisei suishin honbuchōo自由民主党憲 法改正推進本部長), 10 juillet 2014, Terebi Asabi. Le 20 avril 2015, le même député confirmait qu' « en cas de révision de la Constitution, la conscription pourrait être réintroduite $\gg$. 
propos furent ressortis par l'opposition l'accusant de vouloir remettre en selle la conscription. La ministre avait alors répliqué qu'elle s'était située sur le terrain éducatif et qu'à ce titre cette « incorporation » avait quelque utilité, mais qu'elle n'entendait pas remettre en cause la position officielle du Cabinet sur le service militaire ${ }^{49}$.

Surtout, les arguments en faveur du service militaire sont d'abord d'ordre psychologique et moral : la jeunesse japonaise d'après-guerre a besoin d'être reprise en main après des décennies d'une éducation permissive, sinon gauchiste, qui lui ont fait perdre le sens des « vraies » valeurs morales, du collectif, de la nation. Elle a été gangrenée par l'individualisme et l'hédonisme, un système éducatif fondé sur la primauté des droits sur les devoirs, la déliquescence de l'autorité et du sens de la hiérarchie, le mépris de la chose militaire. Du coup, le Japon se retrouve moralement et militairement vulnérable face à des pays comme la Chine et les deux Corées, surarmés et animés par un esprit revanchard. Ce discours n'est certes pas bien nouveau - il était déjà à l'œuvre dans les années 1980, dans une partie des milieux d'affaires et à la droite du PLD - mais a retrouvé des couleurs à l'occasion des débats autour des nouvelles lois sur la sécurité nationale. Un discours alarmiste et nostalgique, qui épouse parfois les contours d'un révisionnisme, constitutionnel et historique, décomplexé. C'est la raison pour laquelle la nostalgie de la conscription ne doit pas être entendue seulement comme l'instrument qui doit permettre de redonner à la jeunesse le sens de l'État et du sacrifice, mais aussi comme fragment d'un discours dirigé contre les institutions d'après-guerre fabriquées et imposées par l'Occupation américaine ${ }^{50}$. En revanche, depuis les années 1980, la question de la restauration de la conscription s'est elle-même diversifiée en quatre grandes tendances : la première, que l'on peut considérer comme « historique », prône la

49. Josei jishin女性自身, $\mathrm{n}^{\circ}$ du 10 novembre 2015. Madame Inada avait tenu des propos similaires dans le numéro de mars 2011 du mensuel Seiron. Sur cette passe d'armes, Asahi shinbun, 11 octobre 2016, Fukushima Mizuho, vice-présidente du Shamintô, chambre des Conseillers, commission du budget, 11 octobre 2016.

50. Pour un aperçu de ce discours dans les années 1980, en particulier sous l'administration Nakasone, notre étude, Les petits-fils du Soleil. La jeunesse japonaise et le patriotisme, Paris, Presses Orientalistes de France, 1988. Pour un écho plus récent, KakiYa, 1999, p. 195 et $s$. L'auteur est un ancien officier d'état-major des FAD en charge principalement des questions de formation. 
réactivation pure et simple du service militaire : c'est la position en particulier de l'ancien maire-gouverneur très controversé de Tōkyō, Ishihara Shintarō石原慎太 郎; la seconde est favorable à un service national obligatoire qui ne prendrait pas les allures d'un service armé, mais qui serait la contrepartie de l'État-providence à la japonaise : position prônée en particulier par l'écrivaine catholique Sono Ayako 曽野綾子, proche de l'ancien Premier ministre Nakasone Yasuhiro中曽根康 弘 ; la troisième se prononce en faveur d'une remilitarisation de la puissance japonaise et de la détention de forces armées, mais sans passer par la conscription : c'est la position notamment du journal Yomiuri shinbun, qui a le plus fort tirage de l'archipel, exprimée dans ses propres projets de révision de la loi fondamentale de 1994 et de 2004, et qui est finalement la plus proche des thèses du PLD au pouvoir ; la quatrième position, quelque peu hétérodoxe, affichée par exemple par la politologue Miura Ruri三浦 瑠麗, prône la restauration du service militaire afin de promouvoir la paix : les citoyens qui ne connaissent pas le prix du sang à payer et délèguent aux « pros » le soin de faire la guerre sont plus facilement enclins au bellicisme et à abdiquer tout contrôle sur l'appareil militaire et, dans les États démocratiques, les politiciens seront moins tentés de céder aux sirènes nationalistes et aux interventions extérieures sous des prétextes fallacieux si leurs propres enfants étaient davantage exposés ${ }^{51}$.

En toile de fond, un élément commun : la nécessaire sensibilisation de l'opinion aux questions de défense et le « renforcement des assises nationales des FAD ». Une préoccupation récurrente des pouvoirs publics depuis la fin des années 1970. À l'époque, seules 50,4\% des personnes interrogées manifestaient

51. Sur ces différentes prises de positions : pour Ishihara Shintarō, ANN News, 20 juin 2011 ; pour Mme Sono Ayako, Akahata shinbun, 28 septembre 2000, à la suite des recommandations en ce sens d'un organisme privé de conseil du Premier ministre, la

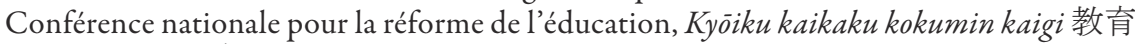
改革国民会議. À noter que Abe Shinzō, en septembre 2006, en campagne pour l'élection à la présidence du PLD, avait développé des idées similaires pour les diplômés des lycées, si l'ensemble des établissements supérieurs devaient aligner la rentrée universitaire sur le mois de septembre : Tōkyō shinbun, 5 octobre 2006 ; MiUra, 2014, p. 188 et s. Elle a été curieusement rejointe par l'essayiste et polémiste Morinaga Takurô 森永卓郎, pour lequel seul le service militaire est de nature à « réveiller » la jeunesse japonaise, à lui faire comprendre les horreurs de la guerre et la dangerosité de la politique actuelle de défense... http://blogos.com/article/86396/. Consulté le 12 août 2016. 


\section{CIPANGO}

302 Cahiers d'études japonaises n²4

de l'intérêt pour les questions de défense, pour 71,5\% en 2015 : un pourcentage jamais atteint dans le passé. Comme l'indiquait, alors, un grand journal japonais à propos de l'Allemagne de l'Ouest : « aujourd'hui, la plupart des Japonais ne sont guère préoccupés des questions de défense. Les prises de position explosives sur la restauration du service militaire doivent faire comprendre que la défense nationale est un problème qui nous concerne tous. C'est ce qui s'est passé en Allemagne fédérale : la population n'avait aucun intérêt pour la défense du pays. Il a donc fallu réviser la Constitution et instituer la conscription pour que chacun se sente ainsi concerné. L'important, ce n'est pas la conscription par elle-même, mais l'institution et ce qu'elle représente ${ }^{52} \gg$. On ne peut mieux résumer la portée et la signification prêtée au service militaire dans le Japon d'après-guerre. Avec cette différence près que l'opinion publique japonaise, comme l'indiquent les sondages, n'est plus aussi partagée ou indifférente sur les problèmes de sécurité nationale : les références iconoclastes à la conscription ne peuvent plus servir, comme par le passé, à « secouer » une conscience nationale endormie par un pacifisme béat. Il n'en est pas moins vrai qu'éveiller, entretenir, approfondir l' « esprit de défense » dans la population reste un objectif important constamment rappelé dans les plans de défense nationale. La menace a sans doute changé de nature depuis la fin de la guerre froide mais, selon le gouvernement, elle est toujours présente avec la montée des tensions régionales, plus diffuse et multiforme avec le terrorisme international. Ce travail de conscientisation est semé d'embûches : si les pouvoirs publics ne peuvent qu'être satisfaits de la hausse du niveau d'intérêt pour les questions de défense, en revanche, la traduction effective de cet intérêt dans l'implication personnelle dans la défense du pays, et notamment celle de la jeunesse japonaise, cas de guerre fait encore difficulté ${ }^{53}$. Quant à la réintroduction de la conscription, elle suscite un

\section{Mainichi shinbun, 7 juillet 1980.}

53. Une enquête internationale menée entre 1989 et 1991 auprès de 37 pays sur les systèmes de valeurs et de croyance indique que le Japon vient bon dernier avec $10 \%$ de réponses favorables à la question suivante : « seriez-vous prêt à vous battre pour votre pays en cas de guerre ? ». Il se situait à 15,2\%, en augmentation, lors de la vague 2010-2014, dont $9,5 \%$ pour les moins de 29 ans. Voir http://www.worldvaluessurvey.org/WVSOnline. jsp. Consulté le 10 juin 2016. Un pourcentage confirmé par un sondage international de la Fondation pour l'innovation politique de 2011 d'après lequel $11 \%$ des jeunes japonais de 16-29 ans se déclaraient prêts « à aller à la guerre et à mourir pour leur pays » : 
rejet massif de la jeunesse du pays, et plus particulièrement des lycéens ${ }^{54}$. C'est la raison pour laquelle des parlementaires conservateurs ont jugé nécessaire un effort spécifique d'information à destination des lycéens, pour souligner que l'adoption du paquet législatif sur la sécurité nationale était nécessaire à la défense du pays, mais n'impliquait pas en aucune façon la réactivation de la conscription ${ }^{55}$.

Dans cette perspective, on comprend mieux les préventions de l'opposition à l'encontre des « lois de guerre » - selon la qualification de l'opposition - adoptées par le Cabinet Abe : l'approfondissement de l'esprit de défense dans un contexte de vulnérabilité régionale, est une forme de «mobilisation idéologique générale de la nation » - une référence datée qui rappelle à dessein le Japon des années 1930 - qui serait les prémisses d'une mobilisation concrète, militaire, rendue plausible par l'autodéfense collective. Le gouvernement japonais a toujours nié en bloc qu'il y ait un quelconque rapport entre l'autodéfense collective et tout retour au service militaire. Il a sans doute raison sur le fond. Mais sa communication a été déficiente à éviter l'amalgame dans lequel s'est engouffrée l'opposition : si, aujourd'hui, les membres des FAD sont davantage exposés au risque, comment

http://www.fondapol.org/en/etudes-en/2011-world-youths/. Consulté le 10 juin 2016. Selon une autre enquête gouvernementale sur les questions de défense, si la volonté de soutenir les FAD en cas d'agression est au plus haut en 2012 avec 56,6 \% de réponses favorables, les Japonais ne sont que $6,8 \%$ à exprimer le souhait de rejoindre les FAD pour participer aux combats, dont 3,7\% des jeunes de la tranche d'âge des 20-29 ans. Un pourcentage stable depuis 40 ans. L'interprétation de ces données doit être prudente : elles expriment peut-être moins la détermination - ou l'absence de détermination - à s'impliquer personnellement dans la défense du pays, que la difficulté à concevoir un rapport à la guerre qui a disparu de la conscience collective après 1945.

54. Selon une enquête rendue publique au printemps 2013 par le syndicat national des enseignants de lycée, le Nikkōkyō 日高教, $63 \%$ des lycéens seraient contre la modification de l'article 9 et 72,5\% contre le retour de la conscription : Akahata shinbun, 20 avril 2013. Pour un état général de l'opinion datant d'août 2015 : sondage des internautes (Yahoo) qui donne $71,5 \%$ de réponses supportant la thèse de l'inconstitutionnalité de la conscription : http://seiji.yahoo.co.jp/article/19/? Consulté le 24 juin 2016.

55. Sankei nyūsu, 28 août 2015. Le journal cite en particulier l'opuscule de Satō Masahisa 佐藤 正久, membre PLD de la chambre des Conseillers et lui-même un ancien des FAD, intitulé Kōkōsei ni mo yondehoshii anzen hoshō no jugyō高校生にも読んでほしい安 全保障の授業 (Cours sur la sécurité nationale à disposition des lycéens) Tōkyō, Wani bukkusu ワニブックス, 2015. 
304 Cahiers d'études japonaises nº 24

garantir que demain la jeunesse japonaise ne sera pas, elle-aussi, appelée à partager ce même risque au nom d'une obligation de défense s'imposant à tous ?

\section{Un palliatif : la « conscription économique »?}

«Si l'on ne s'oppose pas au paquet législatif sur la sécurité nationale, les plus démunis seront contraints de travailler pour les gouvernants $\gg$. Tel fut le propos d'un étudiant de l'université Tōyō (tōyo daigaku 東洋大学) participant à une manifestation devant la Diète japonaise ${ }^{56}$. En octobre 2015, au lendemain de l'adoption des lois sur la sécurité nationale par la Diète japonaise, l'hebdomadaire libéral Kin.yōbi (Kin.yōbi shükan 金曜日週刊) publiait un numéro spécial sur la « conscription économique ${ }^{57}$. Un vocable peu familier au public japonais, mais qui recouvre une autre réalité, ou du moins une possibilité contre laquelle se mobilisent les médias, en tant que forme dégradée et occulte de service militaire. Dans cette perspective, le métier des armes ne serait pas un choix librement assumé, mais déterminé, pour ne pas dire contraint, par la situation économique et financière du pays et la précarisation subséquente de la jeunesse japonaise. Cette « conscription économique » recouvre en réalité trois situations différentes. La première, géographique, n’est pas fondamentalement nouvelle : les bastions de recrutement de la Jieitai, se situent dans les régions du Japon les moins favorisées : les quinze départements les plus pauvres du Japon recrutaient en $200752 \%$ des hommes du rang en provenance du lycée, alors que seuls $27 \%$ des élèves sortant des lycées sont originaires de ces régions. La question reste cependant de savoir s'il existe une politique ciblée ou privilégiée de recrutement en direction de ces zones. La seconde concerne le régime des incitations financières à rejoindre les FAD. C'est ainsi que l'attention des médias a été récemment attirée par un dépliant de l'École de Santé militaire des FAD, Bōei ika daigakkō 防衛医科大 学校, disponible auprès du bureau de recrutement des FAD de Kawasaki川崎 市 (département de Kanagawa), et destiné aux « étudiants dans le besoin » :

56. Dépêche de l'agence Kyōdō, 19 septembre 2015.

57. «Keizaiteki chōheisei ga yattekuru. Wakamono wo sensō ni karu hōhō » 経済 的徴兵制がやってくる。若者を戦争に駆る方法 (Le temps de la conscription économique arrive. Comment forcer la jeunesse à la guerre ), $\mathrm{n}^{\circ} 1059,16$ octobre 2015. 
« Vous rêvez de devenir médecin, infirmier ? Mais vous n'avez pas d'argent : l'École est pour vous ! ». Le dépliant insiste sur les avantages de l'École : une scolarité gratuite - alors que les études médicales comptent parmi les plus chères au Japon - et le statut de fonctionnaire, donc la sécurité de l'emploi ${ }^{58}$. Mais sur les réseaux sociaux, on met plutôt l'accent sur la contrepartie : signer un engagement de neuf ans dans les FAD une fois sorti de l'École; le remboursement des frais de scolarité en cas de départ anticipé et qui peut atteindre 46 millions de yen; la possibilité d'être envoyé en première ligne. D'ores et déjà, il existe un système de bourse des FAD à destination des étudiants de 3ème, 4ème année d'Université et de masters dans les filières médicales, technologiques et scientifiques de l'ordre de 54000 yens mensuels dont le remboursement n'est pas exigé en cas de contrat d'engagement. Ce mécanisme ne concerne actuellement qu'un nombre infime d'étudiants, mais pourrait se développer : depuis juin 2013, le ministère de la Défense étudie en effet de près le système de Reserve Officers Training Corps, en vigueur aux États-Unis ${ }^{59}$. La troisième, liée à la seconde, mais la plus controversée, consiste à « proposer » aux étudiants insolvables, incapables de rembourser leurs aides, des stages dans les services de lutte contre l'incendie, la police et les FAD. Des listes d'étudiants impécunieux auraient été ainsi transmises au ministère de la Défense. Cette proposition avait été faite en mai 2014, alors que le gouvernement s'apprêtait à modifier sa doctrine sur l'autodéfense collective, lors d'une réunion au ministère de l'Éducation sur les aides accordées aux étudiants, par un ancien administrateur des assurances-vie Sumitomo, occupant des fonctions de direction dans la centrale patronale Keizai dōyükai, Maebara Kin.ichi前原 金一, estimant que cette expérience serait enrichissante pour des jeunes sans emploi, et aiderait à leur insertion professionnelle. Le ministère n'avait pas donné suite à cette initiative, mais qui a fini par fuiter dans la presse quelques mois plus $\operatorname{tard}^{60}$, déclenchant une vague d'opposition sur les réseaux sociaux : le Cabinet Abe s'apprêterait à s'aligner sur les pratiques en vigueur aux États-Unis, où le recrutement des forces armées s'effectue surtout auprès des couches défavorisées de la population devenues une chair à canon bon marché. Le gouvernement a toutefois démenti avoir pris aucune

58. Mainichi shinbun, 23 septembre 2015.

59. Akahata shinbun, 2 juillet 2016.

60. Tökyō shinbun, Asahi shinbun, 3 septembre 2014. 
initiative de nature à abonder la thèse de la « conscription économique ${ }^{61} \gg$. Selon des sources proches du ministère de l'Éducation, en 2012, 52,5\% des étudiants étaient titulaires d'une bourse, ce qui représente par diplômé en moyenne 3.000.000 de yens de dette, avec un remboursement de 15000 yens par mois, hors de portée des salariés en CDD, ou en intérim, dont le revenu moyen ne dépasse pas 168000 yens mensuels, contre 473000 yens pour un salarié titulaire, selon les chiffres de l'agence des Impôts nationaux $(2013)^{62}$. Et l'endettement des étudiants est devenu préoccupant : les actions intentées par la JASSO pour défaut de remboursement des bourses seraient passées de 58 en 2004 à 6193 en $2012^{63}$. Par ailleurs, l' « emploi non régulier » représente dorénavant près de $40 \%$ des emplois, et selon les statistiques de la centrale syndicale Rengo 0 連合 (octobre 2014), 76,1 \% des salariés en intérim et en CDD gagnent moins de 2 millions de yens par an. Enfin une autre enquête conduite en 2014 par l'ONG Big Issue Kikin ビッグイシュー基金auprès de jeunes working poors de 20 à 30 ans révèle que $11,5 \%$ d'entre eux ont été, à un moment de leur existence, sans abri. Les jeunes qui n'ont pas décroché le graal du CDI à la japonaise, et rebutés par les CDD et l'intérim, pourraient être ainsi tentés de rejoindre les FAD. Une autre piste a été proposée à la fin 2015 par un ancien chef d'état-major de la région Est : donner aux étudiants qui ne pourraient payer les frais de scolarité élevés de l'université, la possibilité malgré tout de faire des études supérieures, en obtenant par le détour d'un engagement dans la Jieitai, une « qualification » qui leur permettrait d'intégrer l'Université ${ }^{64}$. En novembre 2015, sur proposition du ministère de la

61. Premier ministre Abe Shinzō, chambre des Représentants, réponse écrite $\mathrm{n}^{\circ} 336 \mathrm{du}$ 17 juillet 2015 .

62. http://www.jasso.go.jp/about/statistics/gakusei_chosa/__icsFiles/afieldfile/2015/ 10/16/data12_outline.pdf; https://www.nta.go.jp/kohyo/press/press/2014/minkan/ ; Wakamono no jūtaku mondai.Jūtaku seisaku teiansho [chōsa-hen] 若者の住宅問 題、住宅政策提案書[調査編] (Le problème du logement des jeunes : propositions pour une politique du logement, partie enquête), décembre 2014, bigissue.or.jp/pdf/ teiannsyo2.pdf. Consultés le 25 juillet 2016. Rappelons que les frais de scolarité à l'entrée à l'Université se montaient en 2015 à 817.800 yens pour les universités d'État et pouvaient atteindre jusqu'à 3.392.660 yens pour les universités privées.

63. Tökyō shinbun, 3 janvier 2016.

64. Asahi shinbun, 2 septembre 2015. 
Défense, la section « défense nationale » du PLD a approuvé le principe d'un abattement de 400000 yen sur l'impôt sur les sociétés pour toute embauche d'un réserviste de la Jieitai. Enfin les enquêtes de motivation conduites par le ministère de la Défense tendent à montrer que les motifs économiques, comme en 2009 au lendemain de la crise des subprimes, figurent en bonne place parmi les raisons incitant à intégrer les FAD.

La presse « libérale » constate ainsi que le Japon risque d'emprunter le même chemin que les États-Unis, si au nom de la globalisation, du partenariat transpacifique, le droit du travail japonais ainsi que le système japonais de couverture médicale et sociale devaient être remis en cause ${ }^{65}$. En bref, la relative paupérisation de la jeunesse japonaise, et notamment des étudiants, dans un contexte socio-économique morose constituerait une incitation à embrasser la carrière militaire $^{66}$. L'argument ne va pas à l'encontre de la thèse selon laquelle les lois sur la sécurité nationale pourraient avoir au contraire un effet dissuasif sur le recrutement des FAD : paupérisation et désaffection procèdent d'une rhétorique conjecturale qui n'a qu'un seul objectif : dénoncer la nocivité de la politique globale du gouvernement Abe. De là à soutenir que la « conscription économique » serait une sorte de substitut au service militaire traditionnel, dont les jeunes working poors japonais, et ceux appâtés par des bourses corrélées à un engagement dans la Jieitai, seraient les proies désignées, il n’y a qu'un pas souvent allègrement franchi ${ }^{67}$.

65. Pour des échos dans la presse japonaise du développement potentiel de la conscription économique, Mainichi shinbun, 23 juillet 2015; Ehime shinbun愛媛新聞, 15 septembre 2015 ; Shinano mainichi shinbun 信濃毎日新聞, 28 septembre 2015; Tökyō shinbun, 7 octobre 2015. Également Shükan josei週刊女性 PRIME du 15 septembre 2015 qui évoque le cas des cadres intermédiaires des FAD âgés de 50 ans endettés, chargés de famille, et contraints de ce fait à rester dans la Jieitai. Dans la classe politique, pour le Shamintô, http://www.mizuhoto.org/policy/. En particulier le paragraphe « Policy 01 ». Consulté le 20 août 2017.

66. Ōuchi, 2015, p. 120-124.

67. Tōkyō shinbun, 8 juillet 2015. Pour une position similaire du collectif SEALDs du 6 décembre 2015, https://twitter.com/SEALDs_jpn/status/673383615854546944. Sur le lien supposé entre les campagnes de recrutement des FAD et la « conscription économique », Fukushima Mizuo, chambre des Conseillers, commission spéciale sur 


\section{CIPANGO \\ 308 Cahiers d'études japonaises nº 24}

\section{Conclusion}

Aujourd'hui aucun des partis représentés à la Diète ne préconise la réactivation du service militaire. La plupart des personnalités qui préconisent le retour pur et simple au service militaire restent minoritaires ou ne sont pas, ou plus, en position de responsabilité politique. Au cours du déjeuner régulier avec le bureau de la jeunesse du PLD, le vice-président du Parti, Kōmura Masahiko高村正彦 a, une nouvelle fois, confirmé que la conscription « serait un non-sens politique et militaire ». Il n'en est pas moins vrai que, comme on l'a vu, tant dans la majorité que dans l'opposition, on est loin de camper sur des positions définitives. Pourtant, si le retour généralisé à la conscription ne semble pas en apparence d'actualité, on comprend l'usage politique de la rumeur : du côté de la majorité gouvernementale, elle est instrumentalisée pour discréditer l'attitude de l'opposition et s'en prendre, une fois de plus, au milieu éducatif suspecté de ne pas respecter le principe de neutralité politique. Étrillée par sa défaite aux élections générales de décembre 2012 et 2014 qui laissent entrevoir à la majorité gouvernementale et à ses alliés la possibilité d'une révision constitutionnelle, l'opposition n'avait plus d'autre choix que de faire bloc et de surfer sur les inquiétudes - réelles - de l'opinion en général, et de la jeunesse, en particulier, après l'abaissement de la majorité politique à 18 ans, pour se refaire une santé au moment du renouvellement partiel de la chambre des Conseillers en juillet 2016, et tenter d'obtenir la minorité de blocage pour entraver une éventuelle révision de la loi fondamentale. En agitant ainsi devant l'opinion le chiffon rouge d'une restauration éventuelle du service militaire, l'opposition entendait capitaliser sur la peur de l'avenir, en jouant sur plusieurs registres : celui de l'émotion tout d'abord. Il est assez piquant d'ailleurs de constater qu'elle use exactement du même registre émotionnel et affectif - le devenir des enfants, l'appel aux mères de famille - qu'elle condamnait lorsque le Cabinet Abe en usait et en abusait pour faire passer, auprès d'une opinion publique réticente, les projets de loi de sécurité nationale. Celui ensuite de l'incrémentation graduée : le gouvernement, par petites touches, mettrait en place des procédures, des initiatives et autres politiques publiques, au-delà même de la défense nationale,

les dispositifs juridiques concernant la paix et la sécurité nationale et internationale, 9 septembre 2015. 
balisant la voie vers la restauration du service militaire qui en serait en quelque sorte l'aboutissement logique. Celui de la finalité déductive : ces procédures, initiatives et autres politiques publiques intermédiaires ne font réellement sens qu'en fonction de ce but ultime. Celui enfin de l'objectifflou : la réintroduction de la conscription n'est pas certaine, ni peut-être même souhaitable selon les experts, mais elle reste possible, voire plausible à terme. Compte tenu du caractère éminemment sensible de la question, le Cabinet ne pourrait se permettre d'évoquer publiquement la remise en selle de la conscription, et se trouverait contraint d'adopter une stratégie oblique : dans cette perspective, les démentis officiels ne servent à rien, puisqu'ils viendraient à l'appui de cette politique de dissimulation ${ }^{68}$.

Cette mise en alerte de l'opinion n'a pas été électoralement payante : après avoir accusé un trou d'air au cours de l'été 2015, le taux de soutien au Cabinet s'est sensiblement rétabli au cours du premier trimestre de 2016 et la victoire de la majorité aux élections à la chambre des Conseillers a donné au gouvernement Abe, pour la première fois depuis 1947, une majorité parlementaire arithmétique pour enclencher, le cas échéant, la lourde procédure de révision de la loi fondamentale, et plus particulièrement de la clause de l'article 9. Cette nouvelle donne politique ne change pas, pour le moment du moins, le discours officiel sur l'inconstitutionnalité de la conscription. D'autres observateurs, plus cyniques encore, ajoutent que le service militaire ne sera de toute façon jamais rétabli au Japon car la bureaucratie, les milieux d'affaires, et les politiques refuseront tout net que leurs propres rejetons soient soumis à la conscription... Mais, au-delà des préoccupations politiciennes, l'opposition n'avait pas totalement tort non plus lorsqu'elle a fustigé la volte-face gouvernementale en matière d'autodéfense collective : une interprétation constitutionnelle que l'on croyait consolidée pouvait être remise en cause par des considérations d'opportunité politique. La droitisation certaine du PLD au pouvoir, la concurrence des nationalismes en Asie orientale, la récurrence des tensions régionales donnent consistance à la

68. Ainsi parle-t-on de « conscription larvée 》 (Junchōhei 准徵兵) à propos par exemple de la création en avril 2001 d'une préparation militaire supérieure ouverte aux étudiants de 18 à 34 ans au terme de laquelle ils seraient directement versés dans le cadre de réserve. À bien des égards, la référence à la conscription sert d'épouvantail pour discréditer toute politique visant à faciliter le recrutement des FAD. Pour un exemple de cette rhétorique, Konishi, 2002, p. 54-55. 


\section{CIPANGO}

310 Cahiers d'études japonaises $n^{\circ} 24$

dimension psychologique et symbolique d'une réactivation du service militaire ou d'un service national élargi comme facteur de cohésion nationale. C'est la raison pour laquelle certains militent pour que l'interdiction de la conscription soit sanctuarisée par son inscription explicite dans la loi fondamentale. Mais il faudrait pour cela amender la loi constitutionnelle, et donc ouvrir la boîte de Pandore. À moins d'envisager, comme le préconise l'opposition, que la loi sur les FAD soit modifiée pour que « nul ne soit astreint à rejoindre les FAD contre sa volonté69 ». Mais il est douteux que le Parlement japonais, dans sa composition actuelle, soit prêt à s'engager dans cette voie.

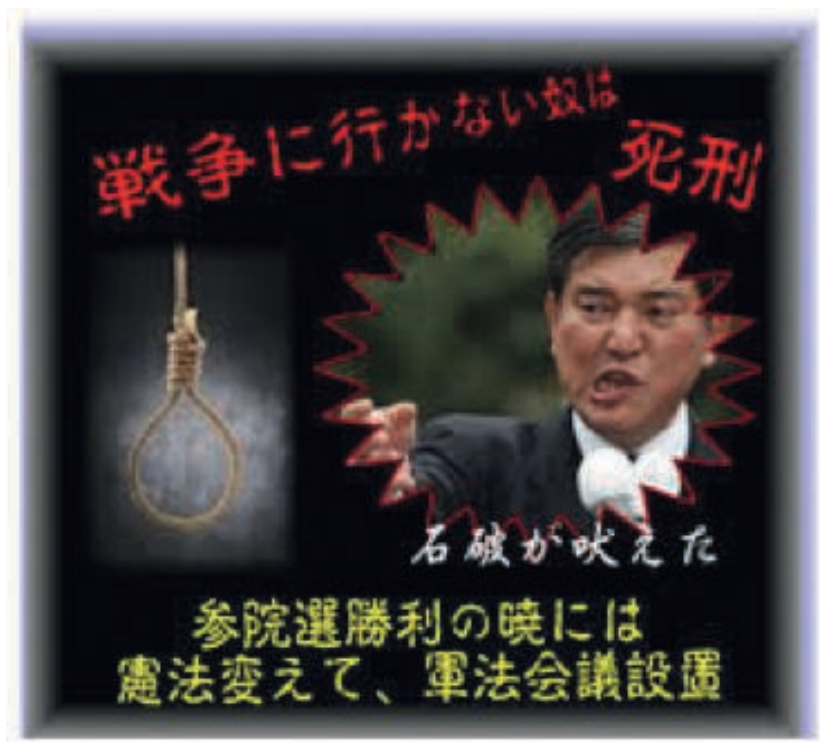

FIGURE 1. POSTER SATIRIQUE FUSTIGEANT LES POSITIONS ULTRA RÉPRESSIVES D'ISHIBASHI SHIGERU.

Source : blogs.yahoo.co.jp

69. Maekawa Kiyoshige, chambre des Conseillers, commission spéciale relative à la législation sur la paix et la sécurité nationale et internationale, 2 septembre 2015. 


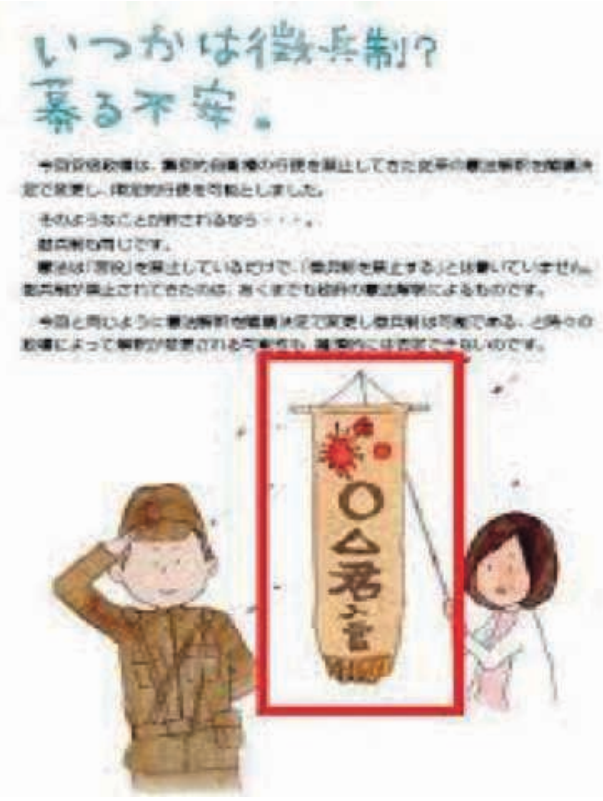

FIGURE 2. POSTER LITIGIEUX DU PARTI DÉMOCRATE DÉNONÇANT LES LIENS SUPPOSÉS ENTRE LES PROJETS DE LOI SUR LA SÉCURITÉ NATIONALE ET LA CONSCRIPTION.

Source : commission d'information du Parti démocrate.

\section{Bibliographie}

FUSE Yūjin布施祐仁, 2015, Keizaiteki chōheisei 経済的徵兵制 (La conscription économique), Shūeisha集英社, Tōkyō, 256 p.

IsHibA Shigeru, 2015, Nihonjin no tame no shüdan jieiken nyümon, 日本人の ための集団自衛権入門 (Introduction à l'autodéfense collective pour les Japonais), Shinchōsha新潮社, Tōkyō, 188 p. 


\section{CIPANGO}

312 Cahiers d'études japonaises n² 24

JASso, 2012, Heisei 24 nendo gakusei seikatsu chōsa kekka no gaiyō 区平成24年 度学生生活調査結果凶の概要, synthèse de l'enquête sur la vie étudiante, exercice, 29 p., URL : http://www.jasso.go.jp/about/statistics/gakusei_ chosa/_icsFiles/afieldfile/2015/10/16/data12_outline.pdf.

KAKIYA Isao 柿谷勲夫, 1999, Chōheisei ga nihon wo sukuu, 徵兵制が日本を救 う (La conscription au secours du Japon), Tendensha 展転社, Tōkyō, 223 p.

KIn.YōBI 金曜日, 16 octobre 2015, « Keizaiteki chōheisei ga yattekuru. Wakamono wo sensō ni karu hō hō. » 経済的徵兵制がやってくる区若者 を戦争に駆る方法, (Comment forcer la jeunesse à la guerre : le temps de la conscription économique arrive), $\mathrm{n}^{\circ}$ 1059, p. 14-25.

KoKUZEICHÔ, 国税庁, agence des impôts nationaux, septembre 2014, Heisei 25 nenbun minkan kyūyo jittai tōkei chōsa kekka ni tsuite平成25年分民間給与 実態統計調査結果について (À propos de l'enquête statistique sur l'état des salaires du secteur privé, exercice 2013), URL : https://www.nta.go.jp/ kohyo/press/press/2014/minkan/

Konishi Makoto 小西誠, 2002, Jieitai no taitero sakusen shiryô to kaisetsu 自 衛隊の対テロ作戦資料と解説 (La stratégie anti-terroriste des FAD, documents et commentaires), 東京, Shakai hihyōsha 社会批評社, Tōkyō, $275 \mathrm{p}$.

Miura Ruri三浦 瑠麗, 2014, « Nihon ni heiwa no tame no chôheisei wo », 日 本に平和のための徵兵制を (Introduire le système de conscription pour garantir la paix au Japon), Bungei shunjû 文藝春秋SPECIAL, vol. 8, $\mathrm{n}^{\circ} 3$, p. 188-191.

NishiKawa Yoshimitsu 西川吉光, 2008, Nihon no anzen hoshō seisaku 日本の 安全保障政策 (La politique japonaise de sécurité), Kōyō shobō 晃洋書房, Kyōto 京都, 301 p. 
ŌE Shinobu大江志乃夫, 2013, Chōhei seido徵兵制度 (L'institution de la conscription), rééd., Iwanami shoten 岩波書店, Tōkyō, 203 p.

Ōuchi Hirokazu大内裕和, octobre 2015, « Hinkonsuru daigakusei to keizaiteki chōhei », 貧困する大学生と経済的徵兵 (La paupérisation des étudiants et la conscription économique), Gendai shisō, 現代思想, sôtokushû anpo hôan wo tou総特集安保法案を問う, numéro spécial de la revue Gendai shisō : les projets de loi sécuritaire en question, vol. 43, n 14, p. 120-124.

SATŌ Masahisa佐藤 正久, 2015, Kōkōsei ni mo yondehoshii anzen hoshō no jugyō高校生にも読んでほしい安全保障の授業 (Cours sur la sécurité nationale à disposition des lycéens), Wani bukkusu ワニブックス, Tōkyō, $191 \mathrm{p}$.

Seizelet Éric, 1988, Les petits-fils du Soleil. La jeunesse japonaise et le patriotisme, Presses Orientalistes de France, Paris, 149 p.

Sōmusho то̄KeIKYoku jinkō suikei chōjiki keiretsu dèta, zenkoku 4 nenrei (kakusai) danjo betsu jinkō (kakunen 10 gatsu tsuitachi genzai) 総務省統計 局人口推計 長時期系列データ 全国 4 年齢（各歳），男女別人 口（各年10月1日現在）, ministère des Affaires générales, direction de la Statistique, données systématiques relatives aux estimations démographiques à long terme, évaluations nationales 4 par âge, répartition par sexe (évaluation annuelle au $1^{\text {er }}$ octobre).

TAKIno Takahiro 瀧野隆浩, 2014, Chinmoku no jieitai. Shirarezaru kunô to benka no 60 nen 沈黙の自衛隊。知られざる苦悩と変化の60年 (Les FAD muettes. 60 ans de souffrances et de changements méconnus), Populasha ポプラ社, Tōkyō, 254 p.

Tamogami Yoshio田母神 俊雄, Nishibe Susumu西部 邁, 富岡 幸一 郎,Tоміока Kōichirō, novembre 2010, Kokumin yo kokubō no gimu ni ōzeyo. Chōheisei wo megutte 国民よ、国防の義務に応ぜよ--徵兵制をめぐっ $\tau$ (Citoyens ! accomplissez vos obligations en matière de défense nationale. À 
314 Cahiers d'études japonaises nº 24

propos de la conscription) (特集 徵兵なき国家は滅びる, numéro spécial : chōhei naki kokka horobiru, Sans la conscription l'État s'effondre, Hyōgensha 表現者, nº33, p. 22-48.

TAmura Shigenobu田村 重信, 2015, Abe seiken to anpo hōsei, 安倍政権と安 保法制 (Le gouvernement Abe et le régime juridique de la sécurité nationale), Naigai shuppan 内外出版, Tōkyō, 272 p.

The Big Issue Foundation, décembre 2014 Wakamono no jūtaku mondai. Jütaku seisaku teiansho [chôsa-hen] 若者の住宅問題、住宅政策提案書[調 査編] (Le problème du logement des jeunes : propositions pour une politique du logement, partie enquête), 40 p., URL : bigissue.or.jp/pdf/teiannsyo2.pdf

YAmaUChI Toshihiro, 2015, Anzen hoshō hōsei to kaiken wo tou, 安全保障法 制と改憲を問う (Questionner la révision de la Constitution et le régime juridique en matière de sécurité nationale), Hō ritsu bunkasha 法律文化社, Kyōto, 249 p.

Les références aux débats parlementaires sont extraites du site suivant de la Diète japonaise : http://kokkai.ndl.go.jp/cgi-bin/KENSAKU/swk_srch. cgi ?SESSION=22830\&MODE $=2$

Résumé : Le « pacifisme constitutionnel », l'anti-militarisme, et le choix de la professionnalisation pour les Forces d'autodéfense japonaises créées en 1954 ont opéré un complet renversement de paradigme dans le mode de recrutement des militaires dans le Japon d'après-guerre. Pourtant, le débat sur le service militaire n'a jamais vraiment disparu de la scène politique. Même si les gouvernements conservateurs ont toujours soutenu que la conscription ne correspondait plus aux besoins des armées modernes et était contraire à la loi fondamentale, la conjonction $\mathrm{du}$ vieillissement de la population et de l'adoption en 2015 du nouveau paquet législatif sur la sécurité nationale risque de compliquer à l'avenir le recrutement des FAD. C'est dans ce contexte particulier que le spectre de la conscription a refait 
surface. Un débat qui ne concerne pas seulement les modalités de recrutement des FAD, mais plus généralement la place du militaire dans la société du Japon d'après-guerre et la « conscientisation » de la jeunesse japonaise aux impératifs de défense nationale.

Mots-clés : Japon d'après-guerre, sécurité nationale, Forces d'autodéfense, recrutement, jeunesse, conscription

Constitutionalpacifism, anti-militarism, and the choice for a professionalized Self Defense Forces (SDF) created in 1954 led to substantial changes in the recruitment of the military in postwar Japan. Nevertheless, the debate over the conscription system has never left the political arena. Although the conservative cabinets have constantly and publicly supported the view according to which the conscription system was now unconstitutional, and no longer fit the needs for a more sophisticated modern army, the conjunction of an ageing population and the recent adoption of a new set of laws on national security is likely to affect, in the future, the breeding ground of the SDF. In this context, rumors and fears about the opportunity of re-introducing the conscription system re-emerged both among political elites and social networks. The heart of the debate not only revolves around the best way to secure a smooth and sustainable recruitment of the SDF but also, more generally, around the place of the military within Japanese society, and it questions the political awareness of the youngest generations on matters related to national defense policy as well.

Keywords: Postwar Japan, national security, Self Defence Forces, recruitment, youth, military service.

キーワード：戦後日本、安全保障、自衛隊、採用、青少年、徵兵 制 